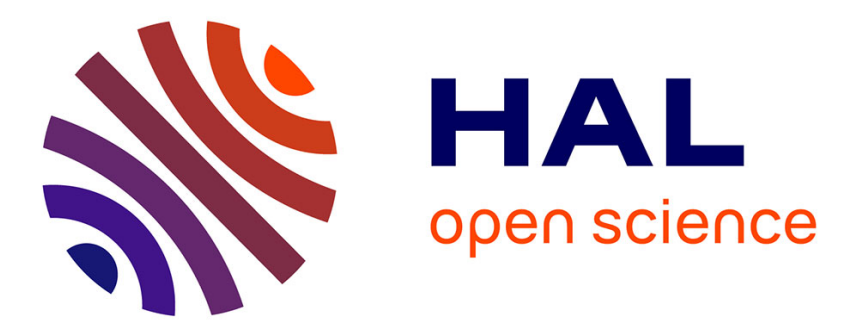

\title{
Les processus de transformation et les perspectives de valorisation des élevages ovins de montagne entre PAC et spécificités locales. L'exemple des Pyrénées Centrales
}

Monique Barrue-Pastor, Claude Lucbert

\section{- To cite this version:}

Monique Barrue-Pastor, Claude Lucbert. Les processus de transformation et les perspectives de valorisation des élevages ovins de montagne entre PAC et spécificités locales. L'exemple des Pyrénées Centrales. Revue de Géographie Alpine / Journal of Alpine Research, 1992, 80 (4), pp.147-166. 10.3406/rga.1992.3656 . hal-02865814

\section{HAL Id: hal-02865814 https://hal.science/hal-02865814}

Submitted on 12 Jun 2020

HAL is a multi-disciplinary open access archive for the deposit and dissemination of scientific research documents, whether they are published or not. The documents may come from teaching and research institutions in France or abroad, or from public or private research centers.
L'archive ouverte pluridisciplinaire HAL, est destinée au dépôt et à la diffusion de documents scientifiques de niveau recherche, publiés ou non, émanant des établissements d'enseignement et de recherche français ou étrangers, des laboratoires publics ou privés. 
Les processus de transformation et les perspectives de valorisation des élevages ovins de montagne entre PAC et spécificités locales. L'exemple des Pyrénées Centrales

Monique Barrué-Pastor, Claude Lucbert

\section{Citer ce document / Cite this document :}

Barrué-Pastor Monique, Lucbert Claude. Les processus de transformation et les perspectives de valorisation des élevages ovins de montagne entre PAC et spécificités locales. L'exemple des Pyrénées Centrales. In: Revue de géographie alpine, tome $80, \mathrm{n}^{\circ} 4,1992$. pp. 147-166;

doi : https://doi.org/10.3406/rga.1992.3656

https://www.persee.fr/doc/rga_0035-1121_1992_num_80_4_3656

Fichier pdf généré le 22/04/2018 


\begin{abstract}
Abstract : The processes of transformation and the prospects for improving mountain sheep rearing between the CAP and local characteristics : the example of the Central Pyrenees. The article analyses the main elements which have marked the transformation of stock-rearing in the Pyrenees, identifying the interrelationships between aspects based on local characteristics, the logic underlying the sheep sector of the market and/or changes in die CAP. The article does not seek to provide a clear illustration of the effects of a policy but radier to analyse the levels of coherence, responsiveness and contradictions that such a policy might have on a mountain area, considered in all its complexity, and on its prospects for the future, and particularly on its ability to upgrade the quality of its products.
\end{abstract}

\title{
Résumé
}

Résumé : Cet article propose une analyse des principaux éléments qui ont marqué le processus de transformation des élevages pyrénéens, en mettant en évidence les interrelations entre ce qui relève de spécificités locales, de la logique du marché de la filière ovine et/ou de l'évolution de la PAC. II s'agit moins d'illustrer concrètement les effets d'une politique que d'analyser les niveaux de cohérence, de résonnances et de contradictions qu'elle peut avoir sur une zone de montagne appréhendée dans sa complexité et ses perspectives d'avenir ; notamment sur ses capacités à valoriser la qualité de ses productions.

\section{Zusammenfassung}

Zusammenfassung : Die Umwand- lungsprozefie und die Perspektivender Inwertsetzung der Bergschafzucht zwischen gemeinsamer Agrarpolitik und lokalen Spezifitäten : das Beispiel der Zentralpyrenaen. Dieser Artikel bietet eine Analyse der wichtigsten Elemente, die den Prozeb der Wandlung der Pyrenäenaufzuchten gekennzeichnet haben in dem er die Zusam- menhângen zwischen lokalen Spezi- fitaten, der Logik des Schafmarktes und/oder der Entwicklung der gemeinsamen Agrarpolitik herausstellt. Es handelt sich weniger darum konkrét die Auswirkungen einer Politik zu illustrieren, als die Ebenen der Zusammenhánge, der Resonan- zen und der Widerspriiche zu analysieren, welche sie auf ein Berg- gebiet haben kann in Bezug auf seine Vielschichtigkeit und seine Zu- kunftsperspektiven, insbesondere seine Fàhigkeit die Qualitát seiner Produktionen in Wert zu setzen. 


\section{Les processus de transformation et les perspectives de valorisation des élevages ovins de montagne entre PAC et spécificités locales. L'exemple des Pyrénées Centrales}

Résumé : Cet article propose une analyse des principaux éléments qui ont marqué le processus de transformation des élevages pyrénéens, en mettant en évidence les interrelations entre ce qui relève de spécificités locales, de la logique du marché de la filière ovine et/ou de l'évolution de la PAC. Il s'agit moins d'illustrer concrètement les effets d'une politique que d'analyser les niveaux de cohérence, de résonnances et de contradictions qu'elle peut avoir sur une zone de montagne appréhendée dans sa complexité et ses perspectives d'avenir ; notamment sur ses capacités à valoriser la qualité de ses productions.

Abstract : The processes of transformation and the prospects for improving mountain sheep rearing between the CAP and local characteristics : the example of the Central Pyrenees. The article analyses the main elements which have marked the transformation of stock-rearing in the Pyrenees, identifying the interrelationships between aspects based on local characteristics, the logic underlying the sheep sector of the market and/or changes in the CAP. The article does not seek to provide a clear illustration of the effects of a policy but rather to analyse the levels of coherence, responsiveness and

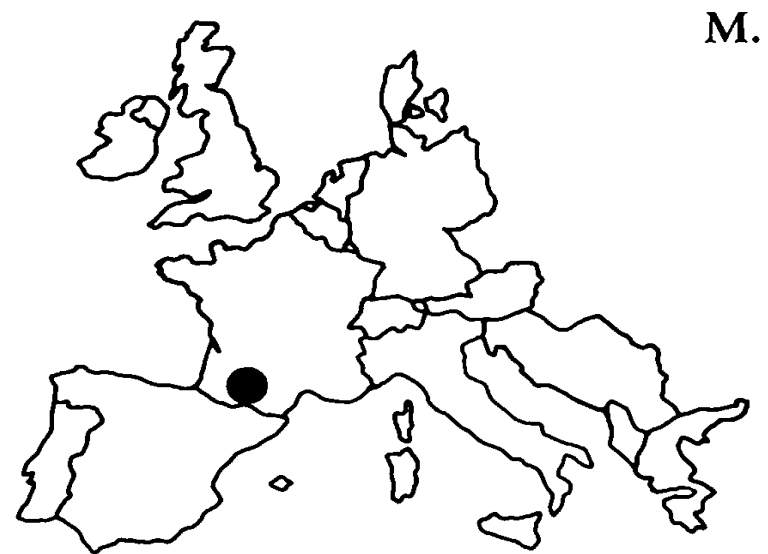

contradictions that such a policy might have on a mountain area, considered in all its complexity, and on its prospects for the future, and particularly on its ability to upgrade the quality of its products.

Zusammenfassung : Die Umwandlungsprozeße und die Perspektivender Inwertsetzung der Bergschafzucht zwischen gemeinsamer Agrarpolitik und lokalen Spezifitäten : das Beispiel der Zentralpyrenäen. Dieser Artikel bietet eine Analyse der wichtigsten Elemente, die den Prozeß der Wandlung der Pyrenäenaufzuchten gekennzeichnet haben in dem er die Zusammenhängen zwischen lokalen Spezifitäten, der Logik des Schafmarktes und/oder der Entwicklung der gemeinsamen Agrarpolitik herausstellt. Es handelt sich weniger darum
1. Cet article a bénéficié de la collaboration de Claude LUCBERT, Directeur de la "Coopérative ovine Garonne-Pyrénéenne" (COGP). 
konkret die Auswirkungen einer Politik zu illustrieren, als die Ebenen der Zusammenhänge, der Resonanzen und der Widersprüche zu analysieren, welche sie auf ein Berg- gebiet haben kann in Bezug auf seine Vielschichtigkeit und seine $\mathrm{Zu}$ kunftsperspektiven, insbesondere seine Fähigkeit die Qualität seiner Produktionen in Wert zu setzen.

\section{$\mathrm{L}_{\mathrm{a}}$} plus sentir, mais elle ne suffit pas à elle seule à expliquer les transformations rapides et fondamentales qui ont touché tous les maillons de la filière ovine au cours de la dernière décennie. Par ailleurs, la problématique de la valorisation des productions ovines de montagne s'est totalement transformée. Vouloir prendre en considération la complexité des éléments, qui interviennent dans le processus de transformation des élevages montagnards, nécessite de mettre en relation la recomposition des structures de commercialisation, la restructuration des groupements de producteurs, les choix politiques locaux et régionaux de

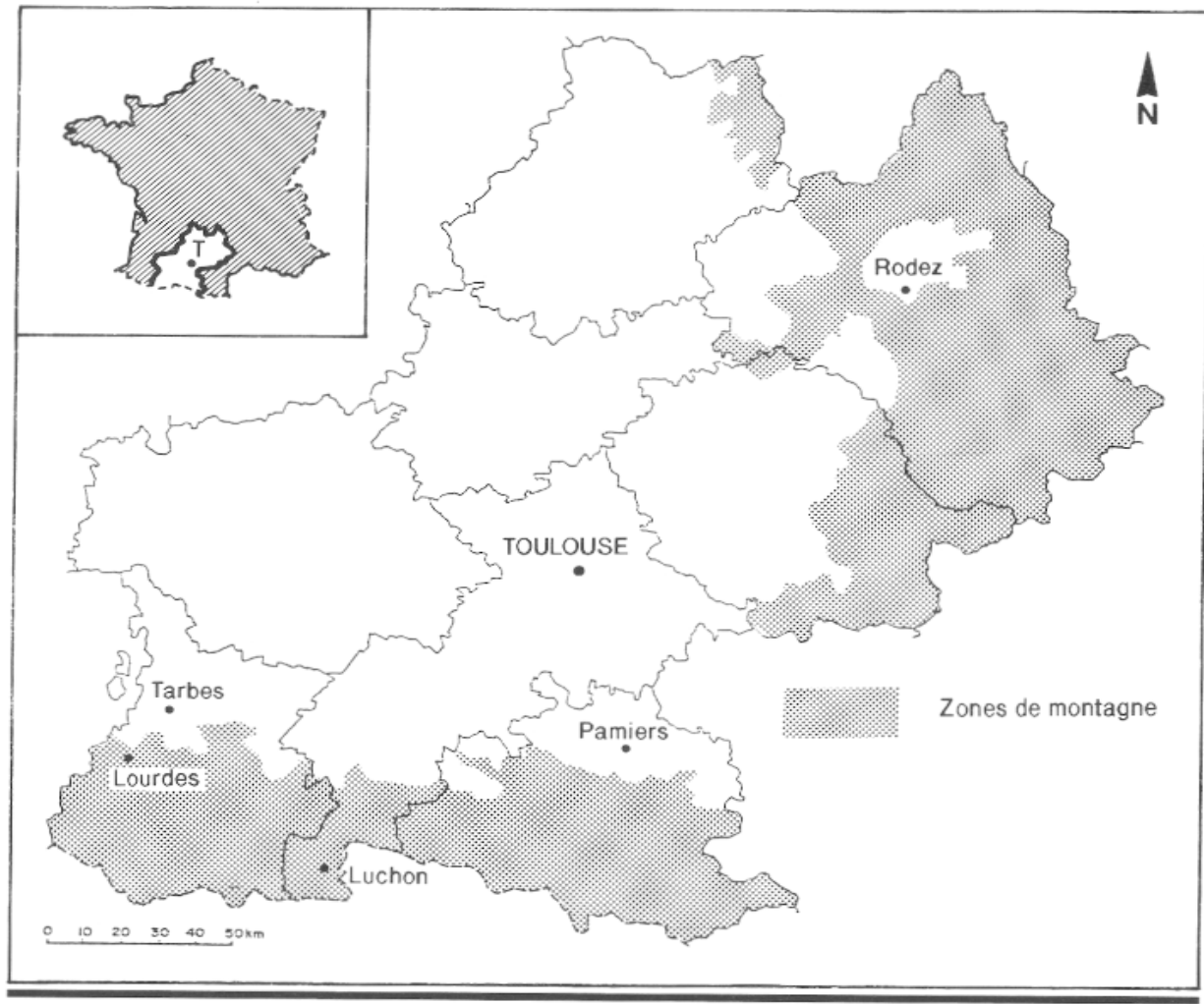

LES PROCESSUS DE TRANSFORMATION ET LES PERSPECTIVES DE VALORISATION... - M. BARRUÉ-PASTOR 
développement de l'élevage avec le choix de contractualisation qui concerne autant les conditions de productions que l'économie de la viande. Seule une analyse fine des principales évolutions enregistrées au niveau régional au cours de ces dix dernières années (ici la Région Midi-Pyrénées et principalement sa zone de montagne) peut permettre d'identifier les différentes interrelations entre ce qui relève de spécificités locales et des tendances "lourdes" issues de la P.A.C. et de la logique de marché et d'émettre quelques hypothèses en fonction de la réforme en cours, sur les perspectives d'avenir de l'élevage ovin dans les Pyrénées.

Les " atouts " et les " contraintes " dévolus à la montagne pyrénéenne laissent une marge de manœuvre limitée en matière de gestion de la fonction productive de l'élevage..., les tactiques d'adaptation ou de résistance, prenant le plus souvent le pas sur de réelles stratégies. L'“ inadaptation " des bâtiments d'élevage (Barrué-Pastor, Barrué, 1991) et des structures foncières héritées, la longueur de l'hiver et les limites des réserves fourragères, l'étroitesse des fonds de vallée et le caractère accidenté des pâturages de la haute montagne... caractérisent les Pyrénées Centrales. A cela viennent s'ajouter les traductions les plus récentes du mouvement social, contenues dans le caractère aléatoire de l'usage des meilleures terres agricoles (héritiers non agriculteurs et multiplication des accords verbaux), la grande hétérogénéité dans la maîtrise technique des systèmes d'élevage (Gibon, 1981), le caractère limité des initiatives individuelles sous le poids des règlements collectifs d'usage des pâturages qui annule les efforts de gestion des calendriers de gestation et des améliorations sanitaires, le poids des agriculteurs âgés sans successeurs et des propriétaires d'animaux sans projet technico-économique (Balent, BarruéPastor, 1986).

Pourtant, les opérations d'aménagement et de développement n'ont cessé de se multiplier ainsi que les efforts d'organisation sous forme coopérative qui ont fini par assurer l'essentiel de la commercialisation des produits de la région (Pugliesi, 1987). Des efforts dont les effets sont restés limités, dans un contexte national et international fort pesant. 


\section{1 - Evolution du marché de la viande euro- péen : résonances en zones de montagne}

Le marché européen de la viande n'a cessé d'évoluer en fonction du règlement qui a été établi en 1980.

Sous l'effet de la compétition communautaire, les élevages se restructurent, dans les Pyrénées, comme dans l'ensemble des zones de montagne de Midi-Pyrénées.

La suprématie de la Grande-Bretagne demeure et l'augmentation galopante de son cheptel continue (il est passé de 26 à 30 millions de têtes entre 1986 et 1989) alors que celui de la France ne cesse de régresser.

L'introduction dès 1986 d'un stabilisateur budgétaire dans le secteur ovin (pourtant déficitaire au niveau communautaire) n'a pas restauré la confiance des éleveurs, pas même la réforme (juillet 1989) des volets internes et externes de l'organisation commune des marchés. Une Q.M.G. (Quantité Maximale Garantie) unique a été définie à $\mathbf{4 4}$ millions de têtes pour l'ensemble de la Communauté. La France qui est le seul pays parmi les douze à voir son cheptel diminuer déplore cette Q.M.G. unique : " un mouton en plus chez nos voisins, c'est un mouton en moins en France ". A chaque dépassement de $1 \%$ du seuil de garantie correspond une réduction de $1 \%$ du prix de base qui sert de référence dans le calcul de la prime compensatrice ovine, qui n'a pas cessée de diminuer depuis 1989.

En 1993, avec l'ouverture du marché unique, il est vraisemblable que les Britanniques ne se contenteront plus d'exporter vers le continent leurs seules marchandises " haut de gamme ". Leur force vient en effet de leur pratique antérieure des " marchés en direct " qui leur permet de maîtriser directement la grande distribution. Ils pourront l'élargir à l'ensemble de leur production, alors que le marché est déjà parvenu à saturation et que la " mauvaise marchandise est invendable » 2 .

Les éleveurs français n'ont alors qu'une seule chance : arriver à produire des agneaux d'excellente qualité. Cela suppose non seulement une mobilisation de l'ensemble de la filière mais aussi une articulation étroite avec les commerciaux, à la fois pour cerner la demande et promouvoir le produit. 


\section{1) Les dictats de la grande distribution}

En bref, deux problèmes essentiels se posent :

- le comportement des partenaires européens (notamment l'Angleterre),

- le comportement de la grande distribution, apatride, qui fonctionne strictement sur les principes et l'anonymat du système bancaire. Elle joue sur les variations des volumes d'approvisionnement à la fois entre zones et entre pays, et induit une restructuration de la filière. En 1980, on importait environ $15 \%$ de la viande ovine consommée en France (évaluée à 240000 t/an); en 1989 on a dépassé le seuil des $50 \%$ d'importation pour atteindre en 1991, $58 \%$. Cette situation n'est pas sans préoccuper " politiques " et " professionnels ".

\section{2) Evolution du négoce français}

La dernière décennie a été marquée par la disparition des petits " chevillards " (c'est-à-dire des petits négociants achetant en vif et assurant l'abattage des animaux) dans les premiers temps, au bénéfice des groupements présents sur les places d'abattage puisqu'ils étaient les seuls fournisseurs; mais il semble que cette situation ne fut que de courte durée. Les clients des petites sociétés de cheville ont rapidement été absorbés par de plus grandes sociétés, puis dans une deuxième phase par des grands groupes (ex : "Centre Sud ") qui se sont eux-mêmes regroupés avec d'autres grandes entreprises (ex : ARCADIE, SOCOPA). Dans ce processus de concentration croissante, les sociétés à dimension régionale ont disparu ${ }^{3}$.

Le caractère de ces groupes est d'être " multi-viandes ", et d'avoir, toujours, un produit " haut de gamme ". Lagneau recherché par la grande distribution a en effet " un maximum de muscle avec un minimum de gras ", avec une certaine image de marque en tant que produit de qualité (Pugliesi, 1987), ce qui, a priori, valorise les races rustiques de montagne mais impose une restructuration des groupements de producteurs.

Trois nécessités leur sont imposées :

- La première est d'être capable de faire du marketing : c'est-àdire savoir miser sur un produit de qualité (ex : agneau primeur) et savoir assurer sa promotion par des campagnes publicitaires.

\footnotetext{
2. Cf. Agri-Midi

(supplément agricole de la Dépêche du Midi),

"Relancer l'élevage ovin", Toulouse, 10 avril 1990 ,

p. 6 .

3. Ainsi en 1980

(De Harting, 198 1), il y

avait en Midi-Pyrénées 17

chevillards dont 12 à

Toulouse. Aujourd'hui il n'y

a que 3 à 4 grandes

sociétés, ce qui crée une situation d'extrême fragilité dans le contexte de concurrence «sauvage» qui existe.
} 
- La deuxième est de s'orienter vers des produits de plus en plus " standardisés ". Il semble que cela soit au prix d'une excellente maîtrise technique que les groupements aient quelques chances de s'imposer sur ce marché.

- La troisième est d'être capable de "vendre en direct " (puisqu'il n'y a plus de chevillards) ; ce qui suppose d'être capable d'abattre soi-même, de se faire référencer, et de livrer. Un nouvel équipement en camions frigorifiques s'impose donc pour pouvoir livrer les carcasses.

Une dernière nécessité semble se dessiner : celle de devoir s'associer à des ateliers de découpe. L'ouverture très récente (à Toulouse) d'un magasin destiné aux grossistes, aux collectivités et aux bouchers semble confirmer cette tendance puisque son objectif est d'arriver à ne vendre que du " piécé " (c'est-à-dire de la viande découpée).

\section{3) La restructuration des lieux d'abattage}

Dans la même période, le règlement européen a imposé de nouvelles contraintes aux structures d'abattage dont l'immobilisme va devenir un frein pour les évolutions rapides en cours. Nombreux sont les abattoirs qui ont connu des difficultés de gestion.

Les normes très strictes établies par la C.E.E. (au niveau sanitaire notamment) ont accéléré l'augmentation des frais, devenus trop lourds pour une même municipalité. Et cela, au moment même où le tonnage traité était en forte régression et où les politiques pratiquées en matière d'investissement semblaient être davantage liées au rythme des élections municipales qu'à celui de la filière viande... Nombreux sont les abattoirs qui ont dû fermer, les plus petits en priorité. Certains vont continuer à exister en tant que "symbole " des abattoirs de montagne (Luchon). Mais ceux des petites villes avoisinantes (Saint-Gaudens, Tarbes, Lourdes et Pau...) sont menacés.

Face à ce mouvement, la tendance est à rechercher une nouvelle rationalité, centrée sur la loi de proximité : proximité entre lieux d'abattage et zone d'approvisionnement, mais aussi entre lieux d'abattage et centres d'engraissement-allotement. 


\section{2 - L'évolution des productions de mon- tagne}

Dans la même période, les productions issues des élevages pyrénéens se sont profondément transformées.

\section{1) La production de broutard 4}

Elle est devenue très limitée. La plupart des broutards mis en marché aujourd'hui sont issus de gros troupeaux qui hivernent en plaine. Cette production est restée importante parmi les agriculteurs en fin de carrière, qui tiennent à conserver leurs anciennes pratiques, mais surtout qui maîtrisent un réseau d'écoulement bien structuré. Leur clientèle principale reste la population maghrébine contactée essentiellement sur le marché du bourg-centre voisin (Lannemezan) ; elle constitue un réel " créneau de valorisation ", même s'il demeure limité (BarruéPastor, Pugliesi, 1992).

\section{2) L'agneau de bergerie}

Cette production a été rémunératrice durant un certain temps précisément au moment où le broutard n'avait plus de marché. Difficile à maîtriser, elle a été dépassée avec l'ouverture du marché espagnol par la vente d'agneaux beaucoup plus légers ; mais aussi par l'évolution des cours du marché. Les cours " favorables " de janvier à mars ont disparu, ils se sont déplacés d'octobre à février, c'est-à-dire, avant l'arrivée des animaux de Lacaune et des agneaux issus des troupeaux laitiers de l'Aveyron. Tel que l'avait conçu la coopérative (la COGP), l'agneau de bergerie était prévu pour être vendu avant la fin février, c'est-à-dire, à un moment où se trouvent sur le marché, des agneaux de plein air de fin de saison, de mauvaise qualité et à la viande rouge. L'objectif était d'ouvrir à ce moment-là un marché pour un agneau primeur, blanc (Brice, Lucbert, 1980).

Si l'agneau de bergerie a échoué, il est clair aujourd'hui que c'est essentiellement par manque de maîtrise de la conduite des troupeaux, pour des raisons fort anciennes qui n'ont pas pu être encore dépassées. Il s'agit essentiellement du refus de l'exclusion des béliers des troupeaux mis en estive ${ }^{5}$, qui montre bien la permanence du poids des anciennes structures de gestion collective, de-
4. Broutard: agneau d'herbe à la chair rose ayant entre 5 et 8 mois, dont la carcasse en viande pèse $20 \mathrm{~kg}$ environ au moment de la vente.

5. Terme pyrénéen pour désigner les pâturages d'altitude, collectifs ; uniquement utilisés l'été. 
venues un handicap face à l'hétérogénéisation du comportement des éleveurs et aux nouvelles nécessités de la production.

\section{3) "L'agneau espagnol "}

Un nouveau marché s'est ouvert en 1988, suite à la demande des pays méditerranéens (notamment l'Espagne, dans une moindre mesure l'Italie) en agneaux légers de type " rustique ».

Si on considère la situation de la zone sud, où les éleveurs n'ont pas de céréales, pas de stocks fourragers importants et pas de bâtiments, c'est une spéculation intéressante. C'est un produit qui présente deux avantages essentiels : il ne demande pas de travail et il libère de la place en bergerie durant l'hiver. Non seulement il ne nécessite aucune modification des systèmes d'élevages locaux, mais il en valorise les atouts et en esquive les contraintes. Nombreux ont été ceux qui ont fait ce choix sans aucune hésitation, sans "vulgarisation ", tant il était conforme à leur système de vie et de travail.

Deux dangers apparaissaient déjà :

- Le créneau, très porteur actuellement, risque d'être éphémère. Les progrès techniques des éleveurs espagnols sont rapides.

- Ce créneau n'est pas par ailleurs sans dangers : il risque d'être un facteur de dilapidation du bon potentiel génétique des troupeaux " tarasconnais » qui est la race rustique locale la plus valorisée. En effet, dès la première année, certains éleveurs sont tentés de " tout " vendre, sans prendre de précaution : c'est-à-dire sans sélection préalable des agnelles de renouvellement; ce qui à terme porterait un coup fatal au potentiel génétique des troupeaux en race locale pure, précisément ceux qui sont susceptibles de trouver de nouveaux débouchés sur le terrain de la revalorisation des produits de grande qualité.

Une première conclusion se dégage : la production ovine régionale possède de réels atouts, à condition de savoir gérer conjointement à la fois le créneau des agneaux légers, mais aussi celui des circuits de qualité à la recherche des " hauts de gamme " (cf. grande distribution). Pour cela il semble qu'il faille un approvisionnement fiable, régulier et continu en quantité et en qualité. Pour utiliser ces opportunités, les organismes économiques de commercialisation se sont fixés pour objectif d'essayer de tirer parti de la capacité des races locales à produire un type d'agneau jeune, de septembre à fin janvier. Il s'agit là d'un véritable créneau de 
commercialisation où la concurrence avec d'autres régions européennes est faible pour ce type de produit de qualité (BarruéPastor, Pugliesi, 1992).

Tout dépend de la capacité des éleveurs et des structures à raisonner et à modifier leurs pratiques de manière concertée.

\section{3 - Restructuration des groupements de producteurs et de leurs moyens}

1) La restructuration des groupements de producteurs et des associations d'éleveurs est en marche. Ainsi la COGP s'est intégrée à la SARL Pyrénées-ovins juridiquement créée en décembre 1988, c'est-à-dire une structure commerciale régionale, qui regroupe tous les groupements du sud.

Au niveau technique, la coopération s'est dotée dès 1985 d'une structure, le CRIOPYC ${ }^{6}$, dans l'objectif de développer l'insémination artificielle. Cette démarche a été intensifiée par le schéma génétique élaboré depuis deux ans avec l'UPRA ${ }^{7}$ et par le nouveau contrat de plan Etat-Région (1989-1993), qui est un véritable « programme de filière ".

\section{2) Un redémarrage des actions génétiques}

Après une première tentative effectuée dans les années 1980, la coopérative a décidé de redévelopper un travail de sélection génétique, en constituant des troupeaux en race tarasconnaise pure. L'idée sous-jacente est à la fois de miser sur l'insémination artificielle pour accélérer la sélection, mais aussi de récupérer au maximum les "souches" de qualité issues de troupeaux de bons et anciens éleveurs. En deux mots, cumuler pratiques innovantes et richesses des savoir-faire locaux.

Outre une modification sensible dans les conditions d'attribution des aides, la principale conséquence de cette action réside dans la transformation des modes de gestion collective des pâturages et des relations entre la plaine et la montagne. Le nouveau plan ovin a défini des impératifs génétiques qui engendrent une modification de l'organisation de l'estivage et donc de la gestion des groupements pastoraux.

C'est ainsi qu'une nouvelle réflexion au niveau de la vallée s'est mise en place très récemment (vallée d'Oueil), visant à créer une même unité pastorale composée de toutes les propriétés individuelles et communales constituant le " pâturage collectif de sou-
6. Centre Régional d'Insémination Ovine des Pyrénées Centrales, logé au domaine expérimental de I'INRA de Toulouse. 7. UPRA = Unité de Promotion des Races Rustiques. 
lane ${ }^{8}$. Elle serait gérée par une structure unique regroupant plusieurs groupements pastoraux actuels, par l'intermédiaire du syndicat intercommunal de la vallée.

Cette situation peut être un facteur de relance des relations entre la plaine et la montagne. En effet, le réseau des exploitations en suivi génétique est constitué de troupeaux tarasconnais en race pure, qu'ils soient situés en plaine ou en montagne. Etant soumis aux mêmes exigences de conduite, l'estivage en commun semble ne plus poser problème, au contraire il permettrait de limiter les frais des uns et des autres.

Quoi qu'il en soit le fait le plus marquant de la décennie 80 reste cette double sélection des troupeaux et des éleveurs.

Certes un gros travail a été effectué en profondeur ; des structures économiques ont été créées, des protocoles de développement élaborés, des marchés conquis, mais il semble que cela ne suffise pas ; il faut aussi " savoir vendre la qualité, à Paris et ailleurs "... 9 Une réelle politique de marketing reste encore à élaborer; les premiers efforts qui ont cherché à imposer l'agneau "Gascogne-Pyrénées " sur les grandes tables parisiennes (notamment avec la complicité du restaurateur André Daguin) semblent prometteurs. Certes, "le Grand Louvre " ouvert sous la pyramide propose chaque jour de l'Agneau des Pyrénées, mais de nouveaux correspondants capables de commercialiser ce produit haut de gamme restent à trouver. Il ne suffit pas de prôner la "qualité ", encore faut-il trouver des acquéreurs qui acceptent de la payer, ce qui n'est pas encore le cas.

\section{4 - Premières réactions à la réforme de la PAC et inquiétudes face à l'échéance de 1993}

8. "Soulane" = versant exposé au Sud, dans les Pyrénées.

9. cf. interview de C. Lucbert (Directeur de la COGPI par M. Barrué Pastor-Mars 1991.
Au moment où l'arme alimentaire redevient un instrument de conquête de pouvoirs et de territoires..., en France et en Europe, on freine "l'acte de produire". Le secteur ovin est certainement le plus en difficultés, alors que (et parce que ?) c'était le plus en expansion. Le règlement communautaire de 1980 n'est certainement pas la seule cause du déclin de l'élevage ovin français, mais il y a beaucoup contribué en précipitant les éleveurs, longtemps abrités par la réglementation nationale, dans une organisation favorisant ouvertement les principaux concurrents (entre autres en prenant comme prix garanti, le niveau de prix français, alors que 


\begin{tabular}{|c|c|c|c|c|c|c|c|c|}
\hline 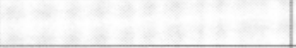 & 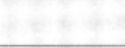 & $V_{0}$ & lume & 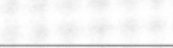 & 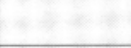 & Prix & els $(* *)$ & \\
\hline 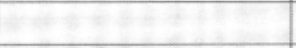 & $70-83$ & $83-88$ & $88-89(1)$ & $89-95(2)$ & $40-83$ & $83-88$ & $88-89(1)$ & $89-95(2)$ \\
\hline Céréales................. & 3,4 & 2,2 & 4,7 & 2,5 & $-2,1$ & $-6,4$ & $-4,0$ & $-4,5$ \\
\hline Oléagineux............ & 7,2 & 23,2 & $-14,0$ & 4,2 & $-1,5$ & $-9,4$ & 14,0 & $-2,5$ \\
\hline Betteraves.............. & 4,1 & $0,7\left(^{*}\right)$ & $-7,0$ & 3,0 & $-2,3$ & $-3,1$ & 3,0 & $-3,0$ \\
\hline Vin......................... & 2,6 & 4,0 & 2,0 & 0,8 & $-2,0$ & $-1,5$ & 17,0 & $-1,3$ \\
\hline Fruits ef légumes... & 1,1 & 1,6 & 1,3 & $0,0(*)$ & $-1,6$ & $-3,8$ & $-1,0$ & $-0,5$ \\
\hline Lait...................... & 2,3 & $-1,6$ & $-2,0$ & 0,0 & $-1,4$ & $-1,5$ & 1,0 & $-1,0$ \\
\hline Viande bovine........ & 1,9 & $0,7\left(\left(^{*}\right)\right.$ & $-4,5$ & $0,0\left({ }^{*}\right)$ & $-2,0$ & $-3,4$ & 6,0 & $-1,0$ \\
\hline Viande ovine.......... & 3,3 & $-3,5$ & $-2,0$ & 2,7 & $-2,7$ & $-6,4$ & $-4,0$ & $-3,0$ \\
\hline Porc...................... & 1,3 & 2,4 & $-1,0$ & $0,0\left(^{*}\right)$ & $-3,5$ & $-8,0$ & 22,0 & $-3,0$ \\
\hline Volaille................ & 5,7 & 3,2 & 7,0 & 4,9 & $-3,4$ & $-4,2$ & $-5,0$ & $-3,0$ \\
\hline CEufs....................... & 2,3 & $0,0\left(^{*}\right)$ & $-4,0$ & 2,1 & $-3,2$ & $-5,8$ & 4,0 & $-3,0$ \\
\hline TOTAL....... & 2,3 & 2,0 & $-0,5$ & 1,5 & $-1,7$ & $-2,2$ & 1,8 & $-2,2$ \\
\hline
\end{tabular}

l'Angleterre était $25 \%$ en dessous et bénéficiait d'un schéma de race organisé, garant d'une production régulière, homogène et à bas prix).

Les aménagements négociés en 1989 modifient quelque peu la situation, mais la commission tire aujourd'hui la conséquence d'un stabilisateur inefficace et d'un " budget communautaire incontrôlable et incapable d'assurer le maintien du revenu des éleveurs " 10 . La crise du marché ovin est maintenant ancienne et la chute des prix continue, malgré tous les efforts qui ont été développés ( $C f$. Tabl. 1).

Non seulement les actions conduites pour développer une politique de qualité et réduire les charges sont insuffisantes, mais elles semblent engendrer des effets pervers en l'absence d'une réelle organisation du marché qui offre aux éleveurs des perspectives d'avenir.

La politique de qualité, affichée au premier plan de la vitrine de la Réforme de la PAC semble totalement illusoire (indépendamment de sa capacité à attirer les subventions) en l'absence de contrôle efficace (au niveau douanier et vétérinaire) des viandes importées, dont l'origine reste floue, mais aussi de critères d'identification de cette qualité par le consommateur. Par ailleurs, cette politique risque d'être remise en cause par d'autres mesures contenues dans la même réforme, entre autres par la multiplication des primes à la brebis et non aux produits.

En effet, les aides directes aux revenus sous forme de primes à la brebis ne cessent de se multiplier et de provoquer de nouvelles inquiétudes ${ }^{11}$.

\section{Tableau 1}

Tendances de la production et des prix à la production à moyen terme (taux de croissance annuel moyen, en \%)

$\left({ }^{*}\right)$ Non significatif (cycle)

$\left.{ }^{* *}\right)$ Déflatés par le prix du PIB

(1) Prévisions de la Direction de la prévision d'Octobre 1989

(2) Prévisions du modèle "Magali", Février 1989

10. Cf. Lovis Mermaz BIMA - nov. 1991.

11.- Il y a eu revalorisation dès 1990 de l'Indemnité Spéciale Montagne (I.S.M.) de + de $14 \%$ par rapport à 1989 , ce qui lui permet d'atteindre un maximum de $910 \mathrm{~F}$. en haute montagne. - La Prime Compensatrice Ovine (P.C.O.l, attribuée ltoutes zones) par brebis, a été légèrement augmentée (142 F. pour 1989). 
- Pour compenser les

« handicaps " des zones de montagne et des zones défavorisées, la commission de Bruxelles a décidé d'une aide spécifique /dite des "Quatre Ecus» de

31 F. par brebis, pour atténuer les effets du stabilisateur.
Les conséquences déjà perceptibles de cette multiplication des primes ne semblent pas conformes à l'objectif de renforcer la production des zones défavorisées qui leur était attribué. Etant distribuées indépendamment des "produits " issus des élevages, elles ont eu une répercussion négative sur les améliorations technico-économiques en cours. Certes, elles ont engendré une augmentation du cheptel, mais aussi une diminution de la production et de sa qualité.

Les éleveurs ont gardé le maximum de brebis, c'est-à-dire, qu'ils n'ont pas "réformé ", pour augmenter les effectifs du cheptel (primé). S'il y a accroissement du cheptel, il y a vieillissement du troupeau (car les agnelles n'ont pas droit à la prime compensatrice).

Parallèlement, les "prix réels " français ne cessent de chuter et vont continuer selon les prévisions ( $c f$. Tabl. 1). Le déficit issu de la chute des cours et de l'augmentation des charges reste difficile à assumer, malgré les primes. A ce rythme, il est légitime de se demander quels sont les producteurs d'ovins qui vont rester ? et avec quel système de production? Tout concourt à penser qu'en montagne ce seront des "propriétaires d'animaux " et non des "éleveurs".

Plusieurs raisons de nature différente convergent dans ce sens : Comme nous venons de l'expliciter, le niveau des primes et des aides attribuées à la brebis est tel que les producteurs se désintéressent des produits. Leur seul souci est de limiter au maximum les frais. Pour des raisons géographiques et conjoncturelles, les éleveurs pyrénéens bénéficient de la proximité du marché espagnol très demandeur en " agneaux légers " depuis plusieurs années; ce qui leur assure l'écoulement régulier d'un produit peu exigeant en charges et en travail. Ce qui a localement anéanti tous les efforts et les expériences pratiqués par les groupements de producteurs pour essayer d'améliorer la valorisation des productions de montagne (sélection, suivi génétique et engraissement notamment). Les éleveurs se contentent pour l'instant de bénéficier de cette rente de situation, qui vient se cumuler avec les primes et leur assure le revenu minimum qu'ils n'espéraient plus. jaillissement direct sur le producteur de montagne, qui devient à nouveau très sollicité (mais sans doute illusoirement favorisé). 


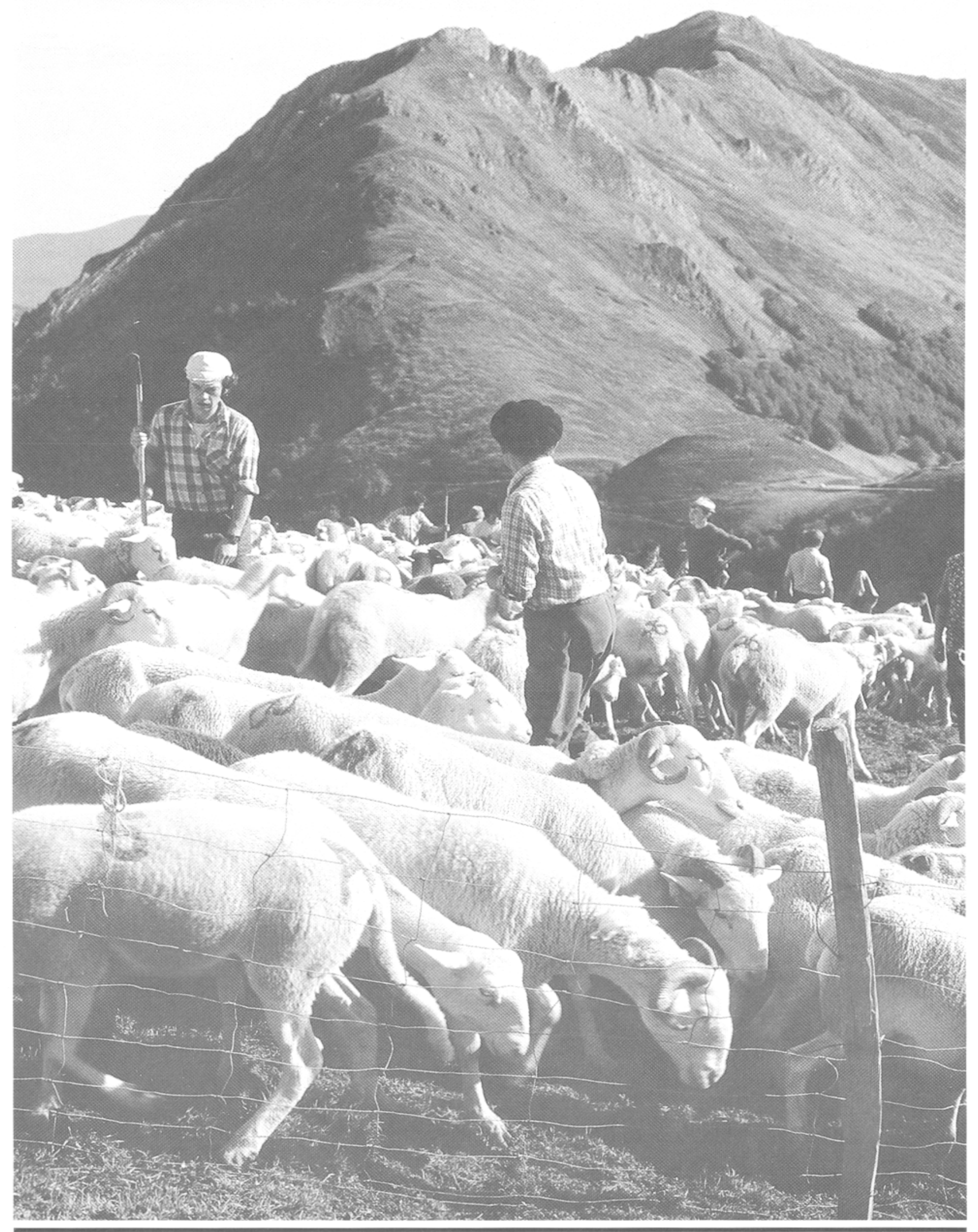


Les zones de montagne connues pour être productrices de " viande maigre ", vont trouver là une manière de valoriser ce qui était considéré jusqu'ici comme un " handicap ».

De nouvelles perspectives apparaissent, émanant de différentes évolutions :

- des élevages intensifs de plaine qui vont se développer avec la chute des cours des céréales et qui vont s'approvisionner en montagne, en agneaux jeunes et légers.

- de la construction d'une nouvelle complémentarité entre plaine et montagne à l'intérieur même des grands groupes coopératifs multi-sectoriels. Cela peut se traduire par la création d'ateliers d'engraissement en relation avec les coopératives céréalières, et cela de deux manières. Soit par la diversification des systèmes de productions céréaliers chez des agriculteurs qui trouvent un complément de revenu dans l'engraissement des agneaux issus de la montagne. Soit enfin par le développement du système d'exploitation propre aux pluri-actifs (qui avait pratiquement disparu, étant devenu trop coûteux) basé sur un hivernage en plaine et sur un pâturage maximum des estives. C'est un retour à la période où les hôteliers étaient propriétaires de brebis, qu'ils ne voyaient que deux fois par an : à la "montée de la plaine " (Gers, Haute-Garonne) pour les confier à un berger d'estive ; puis à la descente des estives et au retour en plaine, au moment où ils les confient à l'agriculteur qui va les soigner durant l'hiver et engraisser les jeunes. Le seul problème est celui de la main-d'œuvre spécialisée qui est nécessaire si l'exploitant n'est pas éleveur ; or les bergers sont de plus en plus difficiles à trouver.

Dans tous les cas, il y a retour vers une conception de la brebis, comme simple " matrice " dont la seule fonction est de donner naissance à des agneaux que l'on cherche à écouler le plus vite possible. Limpression du directeur de la coopérative (COGP) est de vivre une grande régression, un retour vingt ans en arrière, au moment de la création de la coopérative (1970), où il s'agissait de " ramasser " les maigres en montagne pour les engraisser en plaine. De nouvelles "structures de récupération du maigre " vont être créées, les agneaux seront nourris à l'engrais, mais les éleveurs de montagne seront à nouveau "volés " comme ils dans les Pyrénées l'étaient par les négociants et les maquignons. 


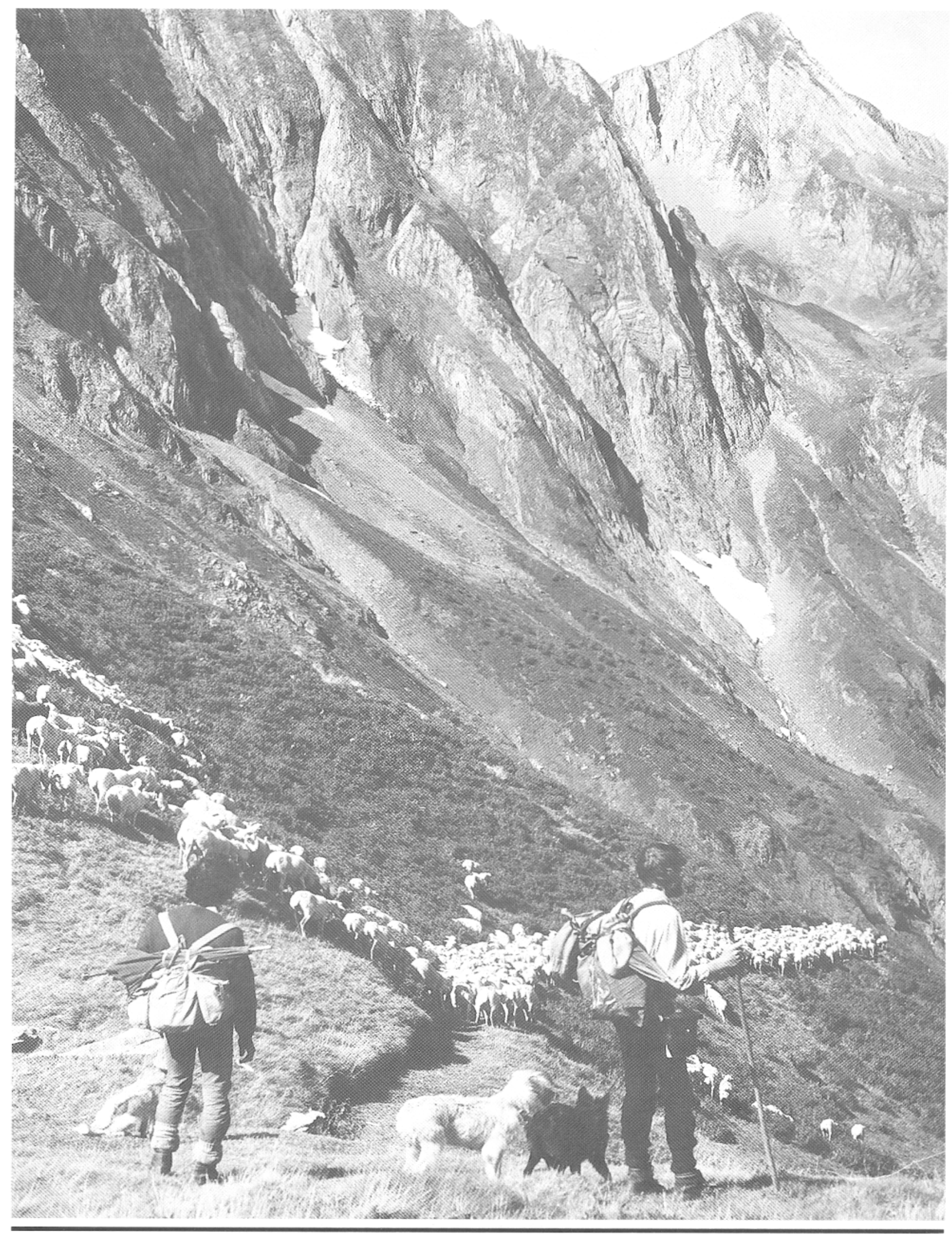


Ce système peu exigeant en travail, en investissement et en charges favorise l'augmentation des effectifs des troupeaux (notamment des pluri-actifs) mais il ne va en aucun cas renforcer la production des zones défavorisées, encore moins leur qualité ; ni favoriser l'installation d'une filière, ni contribuer au bout du compte à l'entretien des espaces montagnards. En effet, les mesures d'accompagnement de la Réforme de la PAC qui prévoient des aides directes pour " pratiques agricoles respectueuses de l'environnement " semblent bien illusoires face à cette tendance des systèmes d'élevage qui va aggraver l'abandon des zones intermédiaires et progressivement la plupart des près de fauche de fond de vallée.

Comment, dès lors, croire en montagne à l'objectif de « renforcer la compétitivité des exploitations en favorisant l'accroissement de la taille des ateliers ovins" ?

Miser sur une augmentation des effectifs de quelques gros éleveurs, alors que les autres disparaîtront, va se traduire par un hivernage systématique en plaine et par la régression de l'entretien de l'espace montagnard. C'est aussi la fin d'une certaine pratique du métier d'éleveur, basée sur une grande maîtrise de la gestion de son troupeau et de son territoire, et dont étaient issus l'essentiel des mâles reproducteurs de qualité. Ces derniers seront de plus en plus difficiles à trouver, pénalisant ainsi tous les efforts génétiques qui ont été réalisés et le développement de l'insémination artificielle. Comment produire dans ces conditions les agneaux de qualité préconisés par la Réforme de la PAC ? Le plan ovin se termine en 1993. Après? C'est le grand inconnu.

Tout aussi inquiétante est la mesure préconisée pour accroître la taille des ateliers ovins : une pré-retraite à 55 ans qui favoriserait l'installation des jeunes sur des exploitations moyennes susceptibles de s'agrandir.

C'est méconnaître le pays, le rapport à la terre et à l'animal. Arrivés à l'âge de la retraite, les exploitants mettent déjà l'exploitation sur le nom de leur femme ; tout laisse supposer que la préretraite ne va qu'aggraver le processus. Contrairement aux effets attendus, elle risque fort de devenir un facteur de blocage supplémentaire à l'installation des jeunes et à l'agrandissement de leur exploitation.

L'importance du total de toutes les primes cumulables à la brebis risque fort d'aller dans le même sens ; elles risquent fort, en effet, de favoriser les propriétaires fonciers, plus que les éleveurs. Il suf- 
fit en effet d'être propriétaire de quelques hectares de terres en montagne (l'accès aux pâturages communaux assurant toutes les garanties complémentaires) pour constituer un troupeau correspondant au maximum d'effectif primable (environ 700 brebis), à condition d'avoir un atelier d'engraissement en plaine.

Un plafonnement de subventions est en cours de discussion avec les Britanniques notamment ${ }^{12}$, dont le comportement reste déterminant dans ce secteur. Comment vont être définis les moyens d'accélérer la restructuration et l'organisation des filières qui restent l'élément déterminant ? Si les tendances à la concentration éliminent toutes les structures régionales de distribution et si les abattoirs proches des zones de montagne continuent à fermer, les perspectives de donner aux éleveurs la possibilité de vivre en montagne demeurent fort aléatoires.

En l'état actuel de la Réforme, tous les dérapages sont possibles. De nombreux " garde-fous " restent à mettre en place si l'on ne ne veut pas que les mesures prises aient l'effet inverse des objectifs affichés.
12. Qui ont déjà obtenu l'intégration de tout leur système de primes dans la Réforme.

13. CIMA : Centre Interdisciplinaire de recherche sur les Milieux naturels ef l'Aménagement rural.

Monique

Barrué-Pastor

CIMA CNRS 13

Toulouse 


\section{Références bibliographiques}

1991 Coll. "Face à la sécheresse et à la crise ovine et bovine " - BIMA - Janvier 1991, pp. 11-17

1992 Coll. (coordination A. Gibon) Les produits ovins de montagne peuvent-ils bénéficier d'une valorisation spécifique?... enseignements de trois opérations dans les Pyrénées Centrales, INRA-CNRS, ITOVIC, 1992, 146 p.

1991 Conseil de l'agriculture : propositions MAC SHARRYBIMA, Juillet 1991-p. 3

CRAMP - Région Midi-Pyrénées Marque régionale: Païs, homologuée comme label agricole - Règlement technique, Toulouse, 22 pages.

1989 Evolution et gestion du monde rural en Europe - Actes du Séminaire, Fondation Universitaire Luxembourgeoise, ARLON, Belgique, 1989

1991 F.A.O. Le marché mondial des viandes - BIMA, Juin 1991, pp. 19-21

1991 La mise en cuvre des mesures socio-structurelles - BIMA, Février 1991, pp. 19-21

1991 La réforme de la PAC: le projet de M. MAC SHARRY Agra-Europe, $n^{\circ} 1,631$, Février 1991

1991 Le mouton français dans l'Europe de demain - BIMA, Février 1991, pp. 7-10

Balent G. 1986 Pratiques pastorales et stratégies foncières dans le Barrué-Pastor $M$. processus de déprise de l'élevage montagnard. in : L'élément et le système — Revue RGPSO, ${ }^{\circ}$ spécial, vol. $57, \mathrm{n}^{\circ} 3,1986$

Barrué-Pastor M. 1991 Architecture, élevage et société en montagne - éd. du Barrué $M$. CNRS, Toulouse, 1991 
Les transformations des produits ovins pyrénéens en rapport avec les transformations de l'économie de marché de la viande, in Les produits ovins de montagne peuvent-ils bénéficier d'une valorisation spécifique ?, INRA-CNRS, ITOVIC, 1992

Pratiques paysannes, logiques commerciales.et rouages institutionnels. L'exemple du Label" "Broutard du pays de Luchon in : Les produits ovins de montagne peuvent-ils bénéficier d'une valorisation spécifique?, INRA-CNRS, ITOVIC, 1992.

Production de broutards dans les élevages ovins du Canton de Luchon (Haute-Garonne), Revue Pâtre $\mathrm{n}^{\circ} 278,1980$, pp. 33-38

La politique agricole commune in : AgriscopeEd. ESA-ANGERS, $\mathrm{n}^{\circ}$ 1, Nov. 1991

Pratiques d'éleveurs et résultats d'élevages dans les Pyrénées Centrales, thèse Docteur-Ingénieur, INA Paris, Grignon, 106 pages, 1981

La commercialisation des viandes ovines et bovines de montagne dans les Pyrénées Centrales, Mémoire de Maîtrise de Géographie, Université de ToulouseLe Mirail, Toulouse, 1981, 400 pages.

Systèmes d'élevages et stratégies de commercialisation des viandes de montagne. L'exemple de trois vallées pyrénéennes: Oueil, Larboust, Barousse, DEA de Géographie, Université de Toulouse-Le Mirail, Toulouse, 1982, 60 pages.

La Réforme de la PAC - BIMA, Paris, 1991, pp. 4-7

Institution et Administration des labels agricoles : la qualité du poulet Label Rouge, Rapport de recherche CEE-DGA, INRA, Toulouse, 1983, 77 pages.
1992 Barrué-Pastor M. Lucbert C.

1992 Barrué-Pastor M. Pugliesi J.P.

1980 Brice G.

Lucbert C.

1991 Carfantan J.Y.

1981 Gibon A.

1981 Harting G.

1982 Harting G.

1991 Mermaz M.

1983 Nicolas F. Sylvander B. 
Pugliesi J.P. 1987 L'impact d'une coopérative sur le développement de l'élevage ovin en zone de montagne : le Luchonnais, Thèse de 3ème cycle de géographie Université de Toulouse-Le Mirail, 1987,350 pages.

Simon B. 1981 La certification de la qualité dans le secteur agroalimentaire, Agriculture n ${ }^{\circ} 449,1981$, pp. $70-74$.

Sylvander B. 1984 La consommation alimentaire : évolutions globales, Lassaut B. différenciations des caractéristiques de produits et des modes de consommation. Le cas du poulet, in : La gestion des ressources naturelles d'origine agricole, pp. 269-395, 1984.

\section{Sigles}

COGP Coopérative Garonne-Pyrénéenne

QMG Quantité Maximale Garantie

CRIOPYC Centre Régional d'Insémination Ovine des Pyrénées Centrales

UPRA Unité de Promotion des Races rustiques 